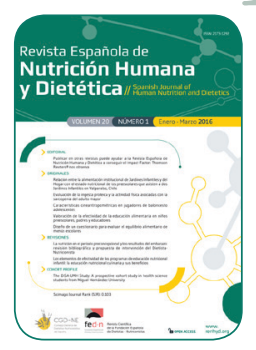

\title{
REVISIÓN
}

\section{La nutrición en el periodo preconcepcional y los resultados del embarazo: revisión bibliográfica y propuesta de intervención del Dietista-Nutricionista}

\author{
Lucia Fernández Molina ${ }^{a}$, José Miguel Soriano del Castillo ${ }^{b, c, d}$, Jesús Blesa Jarque ${ }^{b, c, d, *}$ \\ a Investigadora independiente \\ b Departamento de Medicina Preventiva y Salud Pública, Ciencias de la Alimentación, Toxicología y Medicina Legal, Facultad de \\ Farmacia, Universidad de Valencia, España. \\ C Grupo de investigación CIALBEX (Ciencias de la Alimentación Basadas en la Evidencia y la Experimentación), Instituto \\ Universitario de Ciencia de los Materiales, Parque Científico de la Universidad de Valencia, España. \\ d Unidad Mixta de Investigación en Endocrinología, Nutrición y Dietética Clínica, Universidad de Valencia - Instituto de Investigación \\ Sanitaria HUP La Fe, España. \\ * jesus.blesa@uv.es
}

Recibido el 23 de diciembre de 2014; aceptado el 19 de febrero de 2016.

\section{PALABRAS CLAVE}

Fisiología nutricional durante el embarazo;

Embarazo;

Resultados del embarazo;

Intervención médica temprana;

Intervención dietéticonutricional;

Complementos alimenticios;

Dieta, alimentos y nutrición.
La nutrición en el periodo preconcepcional y los resultados del embarazo: revisión bibliográfica y propuesta de intervención del Dietista-Nutricionista

\section{RESUMEN}

El estado nutricional de la madre antes y durante el embarazo se constata en los últimos años como uno de los factores ambientales más influyentes en el desarrollo fetal. Este estudio se centra en las primeras fases del proceso de gestación o periodo periconcepcional: preconcepción, concepción, implantación, placentación y embriogénesis; puesto que representan un paso crítico en los resultados del embarazo. Tras una revisión bibliográfica exhaustiva de 66 investigaciones publicadas entre los años 1990 y 2015, fueron el zinc, el hierro, el yodo, los ácidos grasos omega-3, el ácido fólico y otras vitaminas del grupo B, los minerales y micronutrientes para los que se han encontrado mayor evidencia de una relación positiva con el desarrollo de la descendencia. Se propone en este estudio el planteamiento de una intervención dietética por parte del Dietista-Nutricionista, tanto individual como colectiva, en el periodo preconcepcional en las mujeres en edad fértil y/o con intenciones de embarazo, pudiéndose continuar dicha intervención en los periodos de gestación, lactancia, postparto e incluso en la descendencia, reportando beneficios a largo plazo y que probablemente se mantengan y manifiesten durante toda la vida del individuo. 


\section{KEYWORDS}

Prenatal Nutritional Physiological Phenomena;

Pregnancy;

Pregnancy outcome;

Early Medical

Intervention;

Early nutritional

intervention;

Dietary

Supplements;

Diet, Food, and

Nutrition.
Preconceptional nutrition and pregnancy outcomes: review and Dietitian-Nutritionist intervention proposal

\section{ABSTRACT}

The nutritional status of the mother before and during pregnancy has been observed in recent years as one of the most influential environmental factors on fetal development. This study focuses on the early stages of gestation or periconception period: preconception, conception, implantation, embryogenesis and placentation; since they represent a critical step in pregnancy outcomes. After an extensive literature review of 66 studies published between 1990 and 2015, iron, iodine, omega 3 fatty acids, folic acid and other B vitamins, minerals and micronutrients for which more evidence of a positive relationship with the development of the offspring were found. The approach of a dietary intervention by the dietitian, both individually and collectively, in the preconception period in women of childbearing age and/or pregnancy intentions is proposed in this study, being able to continue the intervention in periods of gestation, breastfeeding, postpartum and even offspring, causing long-term benefits, which are likely to remain and manifest throughout an individual's life.

\section{CITA}

Fernández Molina L, Soriano del Castillo JM, Blesa Jarque J. La nutrición en el periodo preconcepcional y los resultados del embarazo: revisión bibliográfica y propuesta de intervención del Dietista-Nutricionista. Rev Esp Nutr Hum Diet. 2016; 20(1): 48 - 60. DOI: 10.14306/renhyd.20.1.143

\section{INTRODUCCIÓN}

La dieta y/o el estado nutricional de la madre antes y durante el embarazo se constatan en los últimos años como uno de los factores ambientales más influyentes tanto en el desarrollo fetal como en el potencial reproductivo de la mujer-2-2 así como en el estado de salud de la descendencia ${ }^{3}$.

La mayoría de los estudios donde se analiza el estatus nutricional materno y su implicación en los resultados del embarazo se centran en el segundo y el tercer trimestre por ser una fase crucial en ciertos procesos como el de la organogénesis. Sin embargo, también cabría analizar el papel clave que desempeñan las primeras fases del proceso de gestación o periodo periconcepcional: preconcepción, concepción, implantación, placentación y embriogénesis; puesto que representan etapas críticas para los resultados del desarrollo fetal ${ }^{4}$. A esto se añade el hecho de que un número elevado de mujeres que se quedan embarazadas no son conscientes de ello hasta pasadas las primeras semanas del primer trimestre, periodo en el cual ya se han dado lugar procesos esenciales del desarrollo fetal, por lo que la primera posible intervención ya sería tarde para eliminar determinados factores de riesgo ${ }^{5}$. El número de estudios dedicados a este periodo en concreto es limitado pero se ha producido un incremento en los últimos años gracias a un aumento de la sofisticación de los equipos de ecografía en tres dimensiones $^{6-7}$.

Ahora bien, dentro de este periodo periconcepcional, tiene especial relevancia el periodo preconcepcional, puesto que, si se entiende el embarazo como una serie de etapas que se suceden en el tiempo, los hábitos alimentarios antes de la concepción estarían estrechamente relacionados no sólo con el estado de salud general materno, sino inevitablemente con su capacidad reproductiva2,8.

Por todo ello, ya son varios los autores que aseguran que la dieta y/o hábitos alimentarios deberían estar incluidos en 
los programas de atención en el periodo preconcepcional, o que destacan el diseño en dicho periodo de intervenciones dietéticas específicas encaminadas a la obtención de unos adecuados resultados del embarazo ${ }^{9-15}$.

Debido a la escasez de investigaciones enfocadas al estudio de los patrones dietéticos de forma preconcepcional y su implicación en los resultados del embarazo, se pretende revisar la literatura científica en este aspecto, y puesto que la realización de estos estudios en concreto se describe como una necesidad ${ }^{16}$, la revisión de datos y evidencias científicas al respecto, puede considerarse de gran interés.

Esta revisión bibliográfica también pretende justificar la propuesta de un modelo de intervención del Dietista-Nutricionista (D-N), dado que se ha demostrado que las intervenciones nutricionales en el periodo preconcepcional son efectivas en términos de cambios del comportamiento alimentario por parte de la madre y redundan en un adecuado peso de la descendencia al nacer ${ }^{17}$.

Por todo ello, los objetivos planteados de este trabajo serían: por un lado, esclarecer la relación entre macro y micronutrientes en el desarrollo del periodo preconcepcional y el resultado del embarazo; y por otro lado, la proposición de una intervención dietético-nutricional, individual y/o colectiva, para la mejora del potencial reproductivo y para asegurar un buen estado de salud materno y de la descendencia.

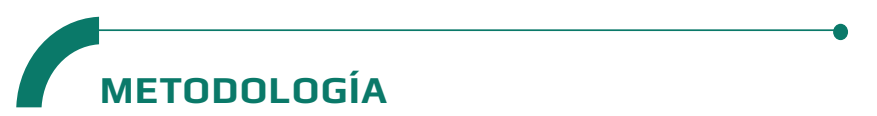

Para la obtención de información de interés perteneciente a datos científicos actualizados se realizó una búsqueda bibliográfica entre octubre de 2012 y noviembre de 2015 identificando estudios publicados en la base de datos electrónica PubMed. Se admitieron múltiples variedades de diseños, entre ellos, estudios experimentales que incluían ensayos clínicos aleatorizados y no aleatorizados, estudios de intervención y observacionales (transversales o longitudinales), estudios de validación de instrumentos, todos ellos tanto en animales como en humanos, e incluso encuestas poblacionales. Dicha búsqueda se llevó a cabo mediante la combinación de conceptos y palabras clave mediante el uso de opciones avanzadas de PubMed, tanto en inglés como en castellano, que aportaban resultados relacionados con el objeto de estudio: nutrición en el periodo preconcepcional y resultados del embarazo. Por tanto, se usaron términos englobados temporalmente en el periodo que precede a la concepción y durante el embarazo temprano ("preconcepción/ preconcepcionalmente", "antes de la concepción", "periconcepción/periconcepcionalmente", "antes del embarazo" o "pre-embarazo"), así como terminología referida a la nutrición ("Índice de masa corporal", "intervención nutricional", "suplementos de micronutrientes", "multivitamínicos y minerales", " zinc ", "ácido fólico", "vitaminas del grupo $\mathrm{B}^{\prime \prime} \mathrm{O}$ "hierro" entre otros). Por último añadir que también se incluyeron conceptos directamente relacionados con la función reproductiva en su globalidad, como son "resultados del embarazo" o "defectos del nacimiento" entre otros similares. Finalmente, se seleccionaron 66 investigaciones publicadas entre los años 1990 y 2015.

\section{MACRO Y MICRONUTRIENTES Y RESULTADO DEL EMBARAZO}

El "éxito" de los resultados del embarazo se traduce como una adecuada sucesión de los procesos de desarrollo tempranos. Por tanto, trastornos o alteraciones como el bajo peso al nacer, defectos del tubo neural, defectos congénitos del corazón, pequeño desarrollo para la edad gestacional, hendiduras oro-faciales, defectos del tracto de salida, diabetes mellitus gestacional, nacimientos a pretérmino o parto prematuro, o abortos espontáneos entre otros, han sido analizados en profundidad para conocer cuáles son los mecanismos específicos de su etiología en los que los nutrientes jugarían un papel esencial. En un reciente estudio retrospectivo de corte transversal Ilevado a cabo en Australia, el cual estaba enmarcado en el periodo preconcepcional, se observó una relación entre los patrones dietéticos en dicho periodo y el nacimiento a pretérmino ${ }^{18}$. Pero si se lleva a cabo un análisis más exhaustivo, sólo en un reducido número de micronutrientes se ha detectado dicho rol o papel y en muchos casos se desconoce el mecanismo biológico por el que la asociación tiene lugar².

Por tanto, a continuación se reflejan los resultados encontrados referentes a sólo un número específico de nutrientes en relación con el embarazo, coincidiendo con los que en la bibliografía científica revisada muestran mayor número de pruebas: el ácido fólico y otras vitaminas del grupo B, el zinc, el yodo, el hierro y los ácidos grasos omega-3. Las principales conclusiones sobre la influencia en humanos de dichos micronutrientes en los posteriores resultados del embarazo, suministrados tanto preconcepcionalmente como en periodos que incluirían el preconcepcional, pueden encontrarse en la Tabla 1. 
La nutrición en el periodo preconcepcional y los resultados del embarazo: revisión bibliográfica y propuesta de intervención del Dietista-Nutricionista

Tabla 1. Resumen con las principales conclusiones sobre la influencia de micronutrientes suministrados antes de la concepción en los resultados del embarazo en humanos.

\begin{tabular}{|c|c|c|c|}
\hline Micronutriente & Trastorno & Resultados & Referencia \\
\hline $\begin{array}{l}\text { Zinc } \\
\text { (Ingesta } \\
\text { preconcepcional) }\end{array}$ & $\begin{array}{l}\text { Defectos del } \\
\text { tubo neural } \\
\text { (DTN) }\end{array}$ & $\begin{array}{l}\text { El riesgo de DTN en los recién nacidos y los fetos } \\
\text { disminuyó con el aumento de la ingesta materna } \\
\text { de zinc. }\end{array}$ & $\begin{array}{l}\text { Velie et al. } \\
(1999)\end{array}$ \\
\hline $\begin{array}{l}\text { Zinc } \\
\text { (Estatus materno) }\end{array}$ & $\begin{array}{l}\text { Nacimiento } \\
\text { prematuro }\end{array}$ & $\begin{array}{l}\text { Pequeña pero significativa reducción del riesgo de } \\
\text { parto prematuro. }\end{array}$ & $\begin{array}{l}\text { Chafee \& King } \\
\text { (2012) }\end{array}$ \\
\hline $\begin{array}{l}\text { Hierro } \\
\text { (Estatus materno) }\end{array}$ & Anemia & $\begin{array}{l}\text { niveles bajos de hierro en la fase } \\
\text { preconcepcional aumentan notablemente el riesgo } \\
\text { de anemia durante el embarazo }\end{array}$ & $\begin{array}{l}\text { Casanueva et } \\
\text { al. (2003) }\end{array}$ \\
\hline $\begin{array}{l}\text { Hierro, magnesio y } \\
\text { niacina. } \\
\text { (Ingesta } \\
\text { preconcepcional) }\end{array}$ & Espina bifida & $\begin{array}{l}\text { Inadecuada ingesta preconcepcional de hierro, } \\
\text { magnesio y niacina asociados con un mayor riesgo } \\
\text { (de } 2 \text { a } 5 \text { veces más) de espina bífida. }\end{array}$ & $\begin{array}{l}\text { Groenen et al. } \\
(2004)\end{array}$ \\
\hline $\begin{array}{l}\text { Hierro } \\
\text { (Ingesta } \\
\text { preconcepcional) }\end{array}$ & $\begin{array}{l}\text { Hendiduras } \\
\text { orofaciales } \\
\text { (HOF) }\end{array}$ & $\begin{array}{l}\text { El aumento de la ingesta de hierro, magnesio, } \\
\text { ácido ascórbico reduce el riesgo de descendencia } \\
\text { afectada por HOF. }\end{array}$ & $\begin{array}{l}\text { Krapels et al. } \\
(2004)\end{array}$ \\
\hline $\begin{array}{l}\text { Hierro } \\
\text { (Ingesta } \\
\text { preconcepcional) }\end{array}$ & $\begin{array}{l}\text { Diabetes } \\
\text { Mellitus } \\
\text { Gestacional } \\
\text { (DMG) }\end{array}$ & $\begin{array}{l}\text { Altos niveles de ingesta de hierro hemo dietético } \\
\text { antes de la concepción pueden estar asociados con } \\
\text { un mayor riesgo de GDM. }\end{array}$ & $\begin{array}{l}\text { Qiu et al. } \\
\text { (2011) }\end{array}$ \\
\hline $\begin{array}{l}\text { Ácidos grasos } \\
\text { omega-3 y omega-6 } \\
\text { (Ingesta } \\
\text { preconcepcional) }\end{array}$ & $\begin{array}{l}\text { Morfología } \\
\text { del embrión. }\end{array}$ & $\begin{array}{l}\text { La ingesta preconcepcional de omega } 3 \text { se } \\
\text { asociaban con la mejora de la morfología temprana } \\
\text { del embrión. }\end{array}$ & $\begin{array}{l}\text { Hammiche et } \\
\text { al. (2011) }\end{array}$ \\
\hline $\begin{array}{l}\text { Ácido Fólico } \\
\text { (Ingesta } \\
\text { periconcepcional) }\end{array}$ & $\begin{array}{l}\text { Defectos del } \\
\text { tubo neural }\end{array}$ & $\begin{array}{l}\text { Hasta un } 72 \% \text { menos de DTN en } \\
\text { la descendencia. }\end{array}$ & $\begin{array}{l}\text { Walker et al. } \\
\text { (2012) }\end{array}$ \\
\hline $\begin{array}{l}\text { Ácido Fólico } \\
\text { (Ingesta } \\
\text { preconcepcional) }\end{array}$ & $\begin{array}{l}\text { Nacimiento } \\
\text { prematuro } \\
\text { espontáneo. }\end{array}$ & $\begin{array}{l}\text { Suplementación de ácido fólico antes de la } \\
\text { concepción durante un año o más: } 70 \% \text { reducción } \\
\text { del riesgo antes de las } 28 \text { semanas de gestación, y } \\
\text { un } 50 \% \text { menos entre } 28 \text { y } 32 \text { semanas. }\end{array}$ & $\begin{array}{l}\text { Bukowski et } \\
\text { al. (2009) }\end{array}$ \\
\hline $\begin{array}{l}\text { Ácido Fólico } \\
\text { (Ingesta } \\
\text { periconcepcional) }\end{array}$ & $\begin{array}{l}\text { Bajo peso al } \\
\text { nacer. }\end{array}$ & $\begin{array}{l}\text { Suplementos suministrados antes de la concepción } \\
\text { se asociaban un valor de } 68 \mathrm{~g} \text { más de peso al nacer } \\
\text { (IC } 37.2,99.095 \% \text { y alrededor de unos } 13 \mathrm{~g} \text { más } \\
\text { en el peso placentario (IC } 1.1,25.595 \% \text { ). }\end{array}$ & $\begin{array}{l}\text { Timmermans } \\
\text { et al. (2009) }\end{array}$ \\
\hline $\begin{array}{l}\text { Ácido Fólico } \\
\text { (Ingesta } \\
\text { periconcepcional) }\end{array}$ & $\begin{array}{l}\text { Defectos del } \\
\text { tubo neural }\end{array}$ & $\begin{array}{l}\text { No encontraron asociación entre el uso de } \\
\text { suplementos de ácido fólico durante el período } \\
\text { periconcepcional y la disminución del riesgo de } \\
\text { defectos de nacimiento, a excepción de los DTNs, } \\
\text { donde dicha asociación sí que se establecía. }\end{array}$ & $\begin{array}{l}\text { Bower et al. } \\
(2006)\end{array}$ \\
\hline $\begin{array}{l}\text { Ácido Fólico } \\
\text { (Ingesta } \\
\text { preconcepcional }\end{array}$ & $\begin{array}{l}\text { Defectos } \\
\text { congénitos } \\
\text { del corazón. }\end{array}$ & $\begin{array}{l}\text { La suplementación preconcepcional de ácido fólico } \\
\text { se asoció con un menor riesgo de } \mathrm{CHD} \text {. }\end{array}$ & Li et al. (2013) \\
\hline $\begin{array}{l}\text { Estado } \\
\text { preconcepcional de } \\
\text { vitaminas del grupo } \\
\text { B }\end{array}$ & $\begin{array}{l}\text { Nacimiento } \\
\text { prematuro o } \\
\text { PP. }\end{array}$ & $\begin{array}{l}\text { El riesgo de parto prematuro fue } 60 \% \text { menor en las } \\
\text { mujeres con niveles suficientes de vitamina B12 } \\
\text { que las que padecían deficiencia, y fue } 50 \% \text { menor } \\
\text { en las mujeres con niveles adecuados de vitamina } \\
\text { B6 que en las que tenían deficiencia. }\end{array}$ & $\begin{array}{l}\text { Ronnenberg } \\
\text { et al. (2002) }\end{array}$ \\
\hline $\begin{array}{l}\text { Vitamina B6 } \\
\text { (Estatus materno) }\end{array}$ & $\begin{array}{l}\text { Concepción, } \\
\text { pérdida } \\
\text { temprana } \\
\text { del } \\
\text { embarazo. }\end{array}$ & $\begin{array}{l}\text { Deficiencia de vitamina B6 reduce la probabilidad } \\
\text { de concepción y contribuyó al riesgo de pérdida de } \\
\text { embarazo en ciclos conceptivos. }\end{array}$ & $\begin{array}{l}\text { Ronnenberg } \\
\text { et al. (2007) }\end{array}$ \\
\hline
\end{tabular}




\section{Ácido fólico}

Unos niveles de folato o ingesta de ácido fólico insuficientes en el periodo periconcepcional junto con factores genéticos y ambientales, se asociaban ya en el 2003 con una serie de defectos de nacimiento ${ }^{19}$. En primer lugar, destacaron los resultados de estudios que analizaron si el adecuado consumo de ácido fólico antes de la concepción podría reducir otros defectos congénitos por su implicación en la vía de la metilación. Dichos estudios asociaron la hiperhomocisteinemia con un consumo inadecuado de ácido fólico ${ }^{20-22}$, siendo dicha alteración la que estaría relacionada con la aparición de defectos congénitos.

Por otro lado, se observó una ligera controversia con la implicación de los folatos en otras alteraciones. En un estudio retrospectivo de cohorte en el cual se elaboró un análisis secundario de los datos referentes a la suplementación preconcepcional de ácido fólico de 34.480 mujeres, se asoció la toma de dichos suplementos con una reducción del 50$70 \%$ de incidencia de nacimiento prematuro espontáneo ${ }^{23}$. En otro estudio retrospectivo de las mismas características (pero desmarcándose ligeramente del periodo estrictamente preconcepcional), los autores a su vez detectaron un ligero aumento del peso al nacer $(68 \mathrm{~g})$ en mujeres suplementadas con ácido fólico durante el periodo periconcepcional (2.274 de 8.880 mujeres) frente a las que no habían recibido la suplementación ${ }^{24}$. Y por último, en un estudio en China que incluía 358 casos y 422 controles se observó que la suplementación preconcepcional de folatos reducía el riesgo de padecer defectos congénitos del corazón, frente a la suplementación que se había producido en la fase gestacional ${ }^{25}$. En contraposición a todo ello, en otro estudio de caso control realizado en Australia, los autores aseguraban que a excepción de los defectos del tubo neural, no existía una relación entre consumo periconcepcional de ácido fólico y otros defectos en el nacimiento ${ }^{26}$. Esta última afirmación podía no ser significativa frente a las evidencias de otros autores dado que la muestra analizada (449 casos y 578 controles) no incluía el análisis de todos los potenciales defectos congénitos, únicamente: hendiduras oro-faciales ( $n=62$ casos), defectos congénitos del corazón ( $n=151$ casos), defectos del tracto urinario ( $\mathrm{n}=117$ casos) y otros defectos ( $n=119$ ). Del mismo modo, determinados autores afirmaban que la suplementación de folatos, a excepción del parto prematuro, no se podía relacionar beneficiosamente con otras complicaciones en el embarazo ${ }^{23}$, pero la discordancia entre investigadores en este caso puede deberse a que estos autores sólo incluían el bajo peso al nacer en la definición de "otras complicaciones" y no fue tratado de forma independiente, de modo que analizaron escasamente la relación entre cantidad de folatos ingerida y peso al nacer, en lugar de comparar el peso al nacer en un grupo de mujeres suple- mentadas con ácido fólico con mujeres sin suplementación, metodología que sí fue llevada a cabo por otros investigadores $^{24}$. Por último, y destacando el aborto espontáneo sobre otras alteraciones, puesto que supone el fracaso del embarazo, otro estudio retrospectivo cuya muestra incluía 11.000 mujeres, con un total de 15.950 embarazos, encontró una reducción del riesgo de un aborto espontáneo en mujeres con altos consumos de ácido fólico antes del embarazo ${ }^{27}$. Ahora bien, respecto a la dosis recomendada, en investigaciones más recientes se ha visto que el consumo de $400 \mu \mathrm{g}$ de ácido fólico al menos 4 semanas antes de la concepción sería suficiente para alcanzar los niveles óptimos de homocisteína y folatos en el plasma necesarios para la prevención de resultados no deseados del embarazo ${ }^{28}$. Esta recomendación coincidiría con la recomendación actual de la Organización Mundial de la Salud: $400 \mu \mathrm{g}$ de ácido fólico desde el momento que se está intentando concebir hasta las 12 semanas de embarazo ${ }^{29}$.

Por último, en una reciente revisión sistemática que analizó la suplementación de ácido fólico en el periodo periconcepcional, los autores confirmaron que la suplementación con ácido fólico previene los defectos del tubo neural y muestra que no hay pruebas suficientes para determinar si el ácido fólico impide otros defectos de nacimiento ${ }^{30}$.

\section{Zinc}

También se han llevado a cabo estudios in vitro, con animales y humanos que analizan la influencia del zinc en los resultados del embarazo y la fertilidad, encontrando todos ellos una asociación positiva entre función reproductora y este nutriente ${ }^{31-39}$, debido principalmente a su implicación en diversas funciones biológicas y rutas metabólicas ${ }^{31}$. Determinados autores mostraron que la deficiencia de zinc, aunque es poco frecuente en países desarrollados, podía ocasionar tanto alteraciones en el feto como malformaciones congénitas ${ }^{31-37}$, aunque sería necesaria más investigación en humanos para establecer esta asociación, ya que la mayoría de estudios eran ensayos in vitro o con animales. Dado que el zinc juega un papel crítico en la síntesis de hemoglobina y en la eritropoyesis, podría desempeñar también un rol significativo en la etiología de la anemia, la cual es indiscutiblemente perjudicial para el embarazo. En un ensayo doble ciego aleatorizado llevado a cabo en Ghana que incluía a 354 mujeres, se estableció que suplementos de hierro y zinc eran más eficaces que una única suplementación con hierro tanto en el aumento de valores séricos de ferritina como hemoglobina en mujeres con deficiencia de hierro en el embarazo, aunque también es cierto que esta relación no se estableció en mujeres con niveles suficientes de hierro ${ }^{38}$. Pero cabe destacar que las 
La nutrición en el periodo preconcepcional y los resultados del embarazo: revisión bibliográfica y propuesta de intervención del Dietista-Nutricionista

deficiencias nutricionales en países en vías de desarrollo suelen producirse de manera multicausal (depleción en el consumo, determinadas enfermedades y otras condiciones relacionados con un estado de carencia de recursos), por lo que resultaría problemático atribuir exclusivamente la disminución de la capacidad reproductiva a una escasez o carencia nutricional concreta ${ }^{40}$ y dichos resultados podrían no ser aplicables a poblaciones con unas características socioeconómicas y/o geográficas diferentes. En otro estudio realizado en California que incluía 430 casos y 429 controles, se encontró una asociación entre la suplementación con zinc de manera preconcepcional y la reducción de la incidencia de defectos del tubo neural, aunque no se aclaró que la reducción del riesgo estuviera correlacionada con el zinc de manera aislada, o una combinación de alimentos 0 nutrientes altamente correlacionados con el zinc ${ }^{39}$.

\section{Hierro}

Otro micronutriente que ha sido ampliamente analizado en la gestación es el hierro, principalmente por su implicación en el desarrollo de anemia durante el embarazo cuando los niveles de ingesta preconcepcional no son óptimos ${ }^{41}$. Asimismo, cabe pensar que si existe una anemia previa al embarazo, ésta podría empeorar tras la concepción, donde los requerimientos de hierro se verán aumentados. En un estudio prospectivo de cohorte llevado a cabo en China que incluía a 405 mujeres, se estableció una relación entre niveles excesivamente bajos de ferritina $(<12 \mathrm{mg} / \mathrm{L})$, así como niveles notablemente altos $(>60 \mathrm{mg} / \mathrm{L})$, ambos en el periodo preconcepcional, y bajo peso al nacer o restricción del crecimiento fetal ${ }^{42}$. Pero cabe destacar que, aunque la evaluación hematológica de determinados índices en el periodo preconcepcional podría ser realmente útil para detectar su asociación con los resultados del embarazo, se ha de tener en cuenta también que la expansión del volumen plasmático durante el embarazo podría afectar a las concentraciones de biomarcadores que hayan sido medidos en varias ocasiones a lo largo del desarrollo fetal, por lo que la interpretación de dichas medidas podría no ser precisa ${ }^{43}$.

Otros trastornos sobre los que se han encontrado hallazgos significativos como consecuencia de una inadecuada ingesta preconcepcional de hierro, serían las hendiduras oro-faciales ${ }^{44}$ y la espina bífida ${ }^{45}$. Pero en ambos estudios de caso-control ( $n=206$ casos y 203 controles para hendiduras oro-faciales; y $n=106$ casos y 181 controles para espina bífida) no se analizó el consumo de hierro de forma individual, sino que se observó el efecto de los niveles bajos de ingestas de varios micronutrientes conjuntamente (magnesio, hierro y ácido ascórbico en hendiduras oro-faciales; magnesio, hierro y niacina en espina bífida) por lo que los efectos obser- vados, derivados de inadecuadas ingestas, podrían no estar relacionados únicamente con este micronutriente, sino que podrían ser un conjunto de consecuencias fruto de varios agentes (nutrientes) causales. Aunque en el estudio acerca de la espina bífida sí se aislaron los valores de ingestas bajas de hierro y se asociaron con la patogénesis de dicho trastorno, no era un estudio diseñado específicamente para dilucidar el papel real que desempeña el hierro, así como tampoco se contempló cuál es el mecanismo de acción por el que dicho micronutriente juega un rol en el desarrollo de la enfermedad.

Por otro lado, en un estudio prospectivo de cohorte de 3.158 mujeres se encontró que altos niveles de ingesta de hierro hemo dietético preconcepcional podían estar asociados con un mayor riesgo de padecer diabetes gestacional ${ }^{46}$, pero en este caso cabría analizar también si los niveles elevados de hierro hemo están ligados a un alto consumo de carnes, vinculado a su vez a un patrón de alimentación menos "saludable" y con menor variabilidad de vitaminas y minerales, el cual se correspondería con el que se describe como "patrón occidental" (rico en carnes, alimentos precocinados y bajo en frutas). Dicho patrón se asoció con menor cantidad de folato en eritrocitos, bajos niveles de vitamina $B_{6}$ y concentraciones de homocisteína elevada ${ }^{15}$, por lo que no sería el perfil de dieta apropiado para mujeres en edad fértil. Asimismo, en otro estudio prospectivo de cohorte que incluía una muestra de 21.411 mujeres, un mayor riesgo de padecer diabetes mellitus gestacional se asoció precisamente con mujeres cuyos patrones alimentarios antes del embarazo consistían en una dieta baja en carbohidratos y alta en proteínas y grasas de origen animal ${ }^{47}$ (similares al citado "patrón occidental"), así como en mujeres con un frecuente consumo de alimentos fritos ${ }^{48}$. Por tanto, surge la necesidad de plantear estudios que analicen la influencia de la ingesta de hierro en la diabetes mellitus gestacional de modo aislado de otras variables como el patrón alimentario.

\section{Ácidos grasos omega 3}

En un estudio prospectivo que analizó la dieta materna de 585 mujeres en el periodo preconcepcional con la probabilidad de aparición de descendencia con pequeño desarrollo para la edad gestacional, se encontró una asociación entre las mujeres que dieron a luz a niños con pequeño desarrollo para la edad gestacional, presentando una menor ingesta de omega 3 y las vitaminas $E$ y $D^{49}$.

En un estudio prospectivo de 235 mujeres donde no se contemplaban únicamente las ingestas dietéticas sino que también se trataban datos hematológicos, se encontró que las mujeres con los niveles más altos de ingestas de ácidos grasos omega 3 en el periodo preconcepcional (en 
particular ácido a-linolénico (ALA) y ácido docosahexaenoico (DHA)) presentaban una mejora del desarrollo y morfología tempranos del embrión ${ }^{50}$.

En un estudio transversal donde se midieron el omega 3 y omega 6 de las membranas de los eritrocitos como biomarcadores, se asoció a las mujeres con un nivel más bajo de omega 3 con alrededor de siete veces más probabilidad a padecer preeclampsia y/o embarazos complicados (en comparación a las que poseían niveles más altos de omega 3). Por ello, los autores consideraban que la ingesta de ácidos grasos omega 3 se debía contemplar como crucial a principios del periodo prenatal, coincidiendo por tanto con el periodo periconcepcional ${ }^{51}$.

\section{Otras vitaminas del grupo B}

En correlación con lo comentado anteriormente acerca de la hiperhomocisteinemia ${ }^{20-22}$, en un estudio de caso-control ( $n=434$ mujeres) realizado en China, se observó que niveles elevados de homocisteína junto con una subóptima ingesta preconcepcional de vitamina $B_{12}$ y $B_{6}$ podía aumentar el riesgo de parto prematuro, de modo que dichos niveles elevados se asociaban con una probabilidad aumentada de casi 4 veces de riesgo de experimentar parto prematuro. El riesgo era $60 \%$ menor en las mujeres sin deficiencia de vitamina $B_{12}$ y un $50 \%$ menor en las mujeres con niveles óptimos de vitamina $\mathrm{B}_{6}{ }^{52}$, por lo tanto, también resultaría de interés realizar estudios para aclarar si la influencia de la homocisteína sobre la aparición del riesgo de padecer algunos trastornos se debe a una acción sinérgica con deficiencias de micronutrientes, o si se ve potenciada por la misma.

Del mismo modo, en otro estudio prospectivo realizado en China que incluía una muestra de 364 mujeres, se encontró una asociación entre un estado deficitario de vitamina $B_{6}$ con un mayor riesgo de pérdida temprana del embarazo ${ }^{53}$. Los autores sugieren que esta asociación podría deberse también a la implicación de la vitamina $B_{6}$ en el ciclo de la homocisteína.

La mayoría de los estudios identificados en esta revisión fueron observacionales, dándose también, aunque en menor medida, estudios de intervención correctamente diseñados. La significación de las conclusiones en las investigaciones fue de aceptable a baja en la práctica totalidad de los resultados, a excepción del efecto beneficioso de la ingesta preconcepcional de ácido fólico en los resultados del embarazo, donde destacaría la disminución del riesgo de incidencia de defectos del tubo neural en la descendencia ${ }^{30}$.

\section{Yodo}

En lugares donde la deficiencia de yodo es grave, el beneficio de la administración de suplementos de yodo durante el embarazo es indiscutible para asegurar el correcto desarrollo del cerebro en el feto ${ }^{54}$, pero en las regiones donde la deficiencia es leve a moderada, la evidencia de beneficio no se encuentra tan ampliamente constatada.

En primer lugar, los autores de un caso clínico, publicado en el 2013, de un recién nacido con defectos del tubo neural y deficiencia de yodo, aseguraban que la suplementación de yodo tanto preconcepcional como en el embarazo era necesaria ${ }^{55}$. Ese mismo año, en una revisión sistemática se ponía de manifiesto el desconocimiento de si los suplementos de yodo (tanto periconcepcionales como prenatales) mejoraban los resultados del desarrollo de la descendencia en ausencia de deficiencia de yodo manifiesta ${ }^{56}$.

La ausencia de evidencias significativas pone de manifiesto la necesidad de más investigación acerca del yodo en el periodo preconcepcional y/o periconcepcional. Aunque, por último, cabría destacar también que en un estudio prospectivo de cohorte que incluía a 168 mujeres, los autores aseguraban que se debería considerar la toma de suplementos de yodo varios meses antes del embarazo, puesto que se encontró un mayor riesgo de insuficiencia tiroidea en mujeres con deficiencia de leve a moderada de yodo que comenzaron la toma de suplementos durante el embarazo en comparación con los que habían usado la sal yodada durante varios años ${ }^{57}$.

En cuanto a la suplementación se refiere, sigue existiendo gran controversia, en primer lugar sobre si las medidas de suplementación universal mediante sal yodada y el consumo adecuado de alimentos ricos en yodo son suficientes para evitar la deficiencia en yodo en la población, y durante el embarazo y la lactancia en particular. En tal sentido discurre el documento de toma de posición del Grupo de Trabajo de Trastornos relacionados con la Deficiencia de Yodo y Disfunción Tiroidea de la Sociedad Española de Endocrinología y Nutrición ${ }^{58}$, donde tras las consideraciones oportunas concluye con la recomendación explicita de la prescripción de yoduro potásico antes de la gestación, si es posible, durante la misma y en el período de lactancia. 
La nutrición en el periodo preconcepcional y los resultados del embarazo: revisión bibliográfica y propuesta de intervención del Dietista-Nutricionista

\section{$\longrightarrow$ PROPUESTA DE INTERVENCIÓN DIETÉTICA}

Por otro lado, y entendiendo el índice de masa corporal (IMC) como un parámetro representativo del estatus nutricional, cabe destacar la asociación positiva del IMC y/o el peso materno medido antes de la concepción, y el peso del recién nacido ${ }^{43,59-61}$ e incluso también que se encontró una relación entre riesgo de padecer un parto prematuro y altos IMC en el periodo preconcepcional ${ }^{62-63}$. Por último, destacar que se ha encontrado una asociación entre el IMC preconcepcional materno con la metilación de genes que participan en el desarrollo de enfermedades crónicas en la descendencia ${ }^{64}$, por lo que la modificación del IMC materno antes de la concepción podría considerarse como un método de prevención de determinadas enfermedades.

Ahora bien, por todo lo comentado hasta ahora y debido a la cantidad de pruebas significativas que se han encontrado, se propone en este estudio la intervención dietético-nutricional en el periodo preconcepcional, pudiéndose continuar en etapas posteriores (embarazo, lactancia, postparto e incluso intervenir en la descendencia) de las mujeres en edad fértil y/o con intenciones de embarazo, con el objetivo de conseguir una mejora del desarrollo del feto, dando lugar por tanto a una descendencia con un buen estado de salud. En el caso del ácido fólico por ejemplo, se ha observado la efectividad de la intervención en lo que a cambios del comportamiento alimentario $y$, por tanto, mejora de los resultados del embarazo se refiere ${ }^{17}$.

La intervención sería llevada a cabo por un profesional sanitario experto en nutrición humana, siendo el DietistaNutricionista la figura idónea para llevarla a cabo puesto que dicha titulación garantizaría que se hayan adquirido las competencias necesarias para tratar los objetivos propuestos satisfactoriamente.

En primer lugar, se llevaría a cabo una evaluación nutricional para conocer el verdadero estatus materno derivado de la ingesta de los distintos macro y micronutrientes. Esta evaluación incluiría a su vez la cumplimentación de una anamnesis y de una historia clínico-dietética: datos personales, historia social, conocimientos previos sobre nutrición, antecedentes familiares, antecedentes patológicos, enfermedades actuales y medicación, y hábitos alimentarios principalmente. Concretamente, para hacer una estimación del perfil dietético materno, las mujeres deberán cumplimentar dos sencillas herramientas de obtención de datos: el Recordatorio 24 horas (R24h) y un cuestionario de frecuencia de consumo validado, los cuales pretenden dar a conocer los valores medios diarios de nutrientes que ingieren las mujeres. En el caso del R24h se efectuaría el registro cuantitativo y cualitativo de su dieta durante siete días (incluyendo el fin de semana). Ambos vendrían acompañados de información complementaria aportada por el profesional de la nutrición que ayudaría al paciente a reflejar de la manera más exacta posible sus hábitos alimentarios (fotos de raciones y otros recursos que el D-N considere adecuados) lo que permitirá poseer unos datos más detallados y/o precisos de los valores de macro y micronutrientes que ingieren. Esta recogida de datos podría ampliarse con un análisis de sangre que determinara la presencia sérica de los nutrientes de interés. Una vez recogida toda la información nutricional se procedería al cálculo de los valores de todos los micronutrientes, añadiendo a cada uno de ellos las cantidades que aportan los suplementos en el caso de que los hubiera, y teniendo siempre en cuenta qué proporción representaban dentro de la dieta en general, por lo que también se calcularía la media de energía que ingiere diariamente cada paciente. Se ha de estimar la ingesta media total de energía de las participantes, ya que no tener en consideración este dato puede alterar la detección de posibles asociaciones entre la ingesta de nutrientes y un determinado proceso patológico general o incluso revertir la dirección de la asociación ${ }^{65}$.

Todo ello se llevará a cabo mediante un programa informático que permita conocer la presencia y proporción de todos los componentes que posee cada uno de los alimentos consumidos, y mediante la adecuación a la cantidad que se estima que ingiere, se obtendrían los valores medios de ingesta de los nutrientes de interés para la intervención, información que se ampliaría con el cuestionario de frecuencia de consumo y el análisis de valores séricos. Posteriormente, dichos valores medios serían comparados con las recomendaciones diarias para las mujeres de su misma edad y condición ${ }^{66}$, y en los casos necesarios se intentaría alcanzar dichas ingestas recomendadas mediante la introducción de cambios en la dieta. En el caso de que la paciente se muestre reticente a modificar su dieta, o que la adhesión a los cambios sea baja, se podría considerar el uso de suplementos minero-vitamínicos, también encaminados al alcance de los valores recomendados.

Aparte, incluso con una correcta ingesta dietética o una buena adhesión a los cambios dietéticos pautados, y tras los datos encontrados en este estudio, sería recomendable la suplementación con ácido fólico y con yodo de forma sistemática en previsión de los graves efectos que puede causar su deficiencia. Además, esta suele ser una práctica clínica habitual y en este sentido existen preparados comerciales adecuados.

Por otro lado, también se llevaría a cabo una evaluación antropométrica, obteniendo valores de peso y altura para conocer el IMC. Estas mediciones se calcularían dada la relación del IMC con la función reproductiva comentada anteriormente ${ }^{59-64}$. 
Por último, destacar que en el momento de modificar la dieta para alcanzar las recomendaciones, sería de vital importancia fomentar la mejora en la educación alimentaria del paciente. Dicha educación se traduce como la adquisición e interiorización de la información y estrategias referentes a la alimentación y a la nutrición que sean necesarias para modificar adecuadamente los hábitos alimentarios de ese individuo. Por este motivo, se destaca la intervención nutricional frente a la suplementación, ya que conseguir la modificación de los hábitos alimentarios significa una estrategia de intervención a largo plazo, dado que los estilos de vida una vez implantados se convierten en "comportamientos" de larga duración. Por el contrario, el uso de suplementos representaría una acción o intervención puntual, ya que los cambios en el paciente serían meramente fisiológicos, sin tener en cuenta el aspecto psicológico e incluso emocional, ni asegurando que dichos cambios permanezcan durante toda la vida del paciente. De este modo, la educación favorecería los resultados de la intervención no sólo en el periodo preconcepcional, sino en las etapas posteriores, como embarazo, lactancia y postparto, llevándose a cabo la misma metodología comentada anteriormente, pero adecuando las recomendaciones a la condición de la paciente.

Por todo ello, con la intención de estandarizar la actuación del D-N en el periodo preconcepcional, se propone un Protocolo de Intervención (Figura 1) a llevar a cabo en mujeres con previsión de embarazo.

Figura 1. Protocolo de intervención dietético-nutricional sobre el periodo preconcepcional en mujeres con previsión de embarazo.

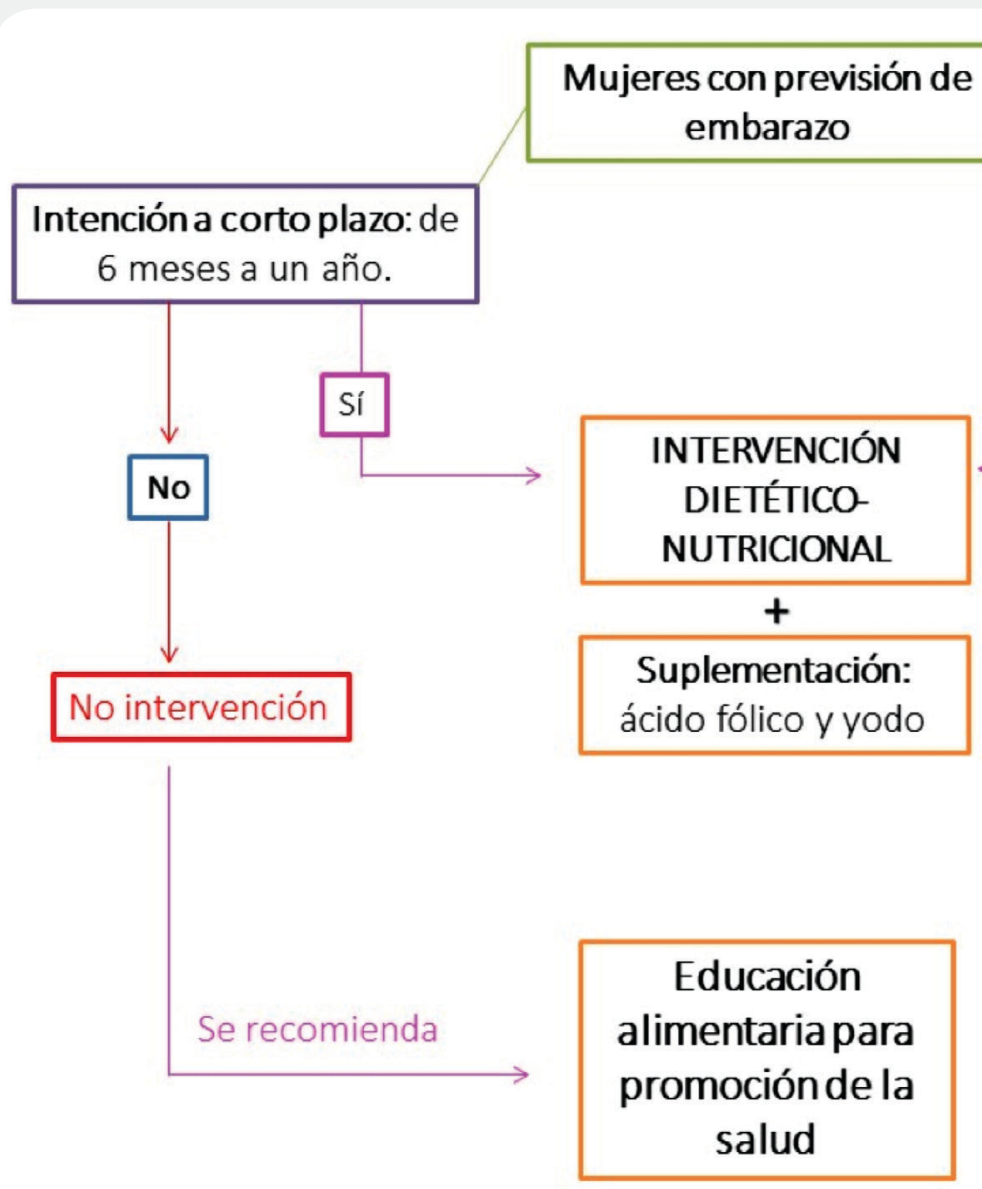


Destacar que también se promociona el uso de la educación alimentaria en detrimento de los suplementos vitamínicos para evitar los posibles efectos no deseados de una "sobresuplementación", puesto que en un estudio retrospectivo que incluía a 35.914 mujeres, se detectó un aumento del riesgo de muerte fetal temprana (menos de 20 semanas de gestación) tras el uso de suplementos multivitamínicos durante el periodo periconcepcional, y especialmente el preconcepcional, aunque dicho uso representaba una disminución del riesgo de muerte fetal tardía (más de 20 semanas) si se continuaba con la toma tras la concepción ${ }^{67}$. Por ello, una intervención más personalizada que incluya dicha educación del paciente sobre su propia ingesta dietética podría perfeccionar los programas de atención en el periodo preconcepcional, encaminándolos a evitar los efectos no deseados de un "exceso" en la ingesta vitamínica a través de los suplementos, puesto que se desconoce el mecanismo biológico por el que producirían estos efectos adversos.

Esta intervención tendría un enfoque preventivo más que funcionar como una protección específica frente a una determinada alteración. Por todo ello, también se proponen campañas de sensibilización en los centros de salud que proporcionen a las mujeres en edad fértil información acerca de unos hábitos alimentarios adecuados y acordes a su situación, dotándoles así de una estrategia más para mejorar su salud y favorecer la capacidad reproductiva y de gestación. Del mismo modo, se podría enfocar tanto la intervención individual como colectiva no sólo a las madres, si no a la descendencia en la etapa más temprana de sus vidas.

Pero aún cabría la realización de más investigaciones en el ámbito de la nutrición y la reproducción humana para así adaptar las ingestas de las mujeres no sólo a las recomendaciones estándar en función de la edad, sino a unos valores de ingestas específicas de cada uno de los micronutrientes involucrados en el potencial reproductivo, y que estuvieran formuladas adecuadamente, de modo que los supuestos efectos beneficiosos de esas ingestas en la fertilidad y reproducción estuvieran contrastados científicamente.

Como conclusión, existen evidencias que demuestran una implicación de determinados nutrientes, ácido fólico, yodo, zinc, hierro y ácidos grasos omega 3 principalmente, tanto en la fertilidad de las mujeres como en el éxito de los resultados del embarazo. Asimismo, la adecuada ingesta de dichos nutrientes debería comenzar antes de la concepción (periodo preconcepcional) para asegurar su efecto beneficioso o al menos impedir que se instaure su deficiencia.

Por otro lado se plantea una intervención dietético-nutricional tanto individual como colectiva de las mujeres que pretenden quedarse embarazadas, con el objetivo de mejorar el potencial reproductivo materno mediante la mejora de su estatus nutricional. Dicha intervención se podría continuar en los periodos de gestación, lactancia, postparto e incluso sobre la descendencia, reportando beneficios a largo plazo que probablemente se mantengan y manifiesten durante toda la vida del individuo.

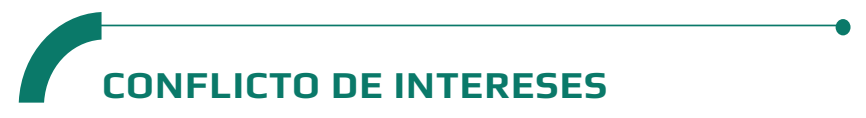

Los autores expresan que no hay conflictos de intereses al redactar el manuscrito.

\section{BIBLIOGRAFÍA}

(1) Ebisch IMW, Thomas CMG, Peters WHM, Braat DDM, Steegers-Theunissen RPM. The importance of folate, zinc and antioxidants in the pathogenesis and prevention of subfertility. Hum Reprod Update. 2007; 13(2): 163-74.

(2) Ramakrishnan U, Grant F, Goldenberg T, Zongrone A, Martorell R. Effect of women's nutrition before and during early pregnancy on maternal and infant outcomes: a systematic review. Paediatr Perinat Epidemiol. 2012; 26(Suppl 1): 285301.

(3) Herman DR, Taylor Baer M, Adams E, Cunningham-Sabo L, Duran N, Johnson DB, et al. Life Course Perspective: evidence for the role of nutrition. Matern Child Health J. 2014; 18(2): 450-61.

(4) Cetin I, Berti C, Calabrese S. Role of micronutrients in the periconceptional period. Hum Reprod Update. 2010; 16(1): 8095.

(5) van der Zee B, de Beaufort I, Temel S, de Wert G, Denktas S, Steegers E. Preconception care: an essential preventive strategy to improve children's and women's health. J Public Health Policy. 2011; 32(3): 367-79.

(6) Rousian M, Koning $A H J$, van Oppenraaij RHF, Hop WC, Verwoerd-Dikkeboom CM, van der Spek PJ, et al. An innovative virtual reality technique for automated human embryonic volume measurements. Hum Reprod. 2010; 25(9): 2210-6.

(7) Rousian M, Verwoerd-Dikkeboom CM, Koning AHJ, Hop WC, van der Spek PJ, Steegers EAP, et al. First trimester umbilical cord and vitelline duct measurements using virtual reality. Early Hum Dev. 2011; 87(2): 77-82.

(8) Chadio S, Kotsampasi B. The role of early life nutrition in programming of reproductive function. J Dev Orig Health Dis. 2014; 5(1): 2-15.

(9) Bhutta ZA, Das JK. Interventions to address maternal and childhood undernutrition: current evidence. Nestle Nutr Inst Workshop Ser. 2014; 78: 59-69. 
(10) Zive MM, Rhee KE. Call to action: continuum of care for females of reproductive age to prevent obesity and ensure better health outcomes of offspring through nutrition. Clin Obstet Gynecol. 2014; 57(3): 446-55.

(11) Cuervo M, Sayon-Orea C, Santiago S, Martínez JA. Dietary and health profiles of Spanish women in preconception, pregnancy and lactation. Nutrients. 2014; 6(10): 4434-51.

(12) Mastroiacovo P, Nilsen RM, Leoncini E, Gastaldi P, Allegri V, Boiani $A$, et al. Prevalence of maternal preconception risk factors: an Italian multicenter survey. Ital ] Pediatr. 2014; 40: 91.

(13) Dean SV, Imam AM, Lassi ZS, Bhutta ZA. Importance of intervening in the preconception period to impact pregnancy outcomes. Nestle Nutr Inst Workshop Ser. 2013; 74: 63-73.

(14) Bhutta ZA, Das JK, Rizvi A, Gaffey MF, Walker N, Horton S, et al. Evidence-based interventions for improvement of maternal and child nutrition: what can be done and at what cost? Lancet. 2013; 382(9890): 452-77.

(15) Vujkovic M, Ocke MC, van der Spek PJ, Yazdanpanah N, Steegers EA, Steegers-Theunissen RP. Maternal Western dietary patterns and the risk of developing a cleft lip with or without a cleft palate. Obstet Gynecol. 2007; 110(2 Pt 1): 37884.

(16) Hambidge KM, Krebs NF, Westcott JE, Garces A, Goudar SS, Kodkany BS, et al. Preconception maternal nutrition: a multisite randomized controlled trial. BMC Pregnancy Childbirth. 2014; 14: 111.

(17) Temel S, van Voorst SF, Jack BW, Denkta S, Steegers EAP. Evidence-based preconceptional lifestyle interventions. Epidemiol Rev. 2014; 36: 19-30.

(18) Grieger JA, Grzeskowiak LE, Clifton VL. Preconception dietary patterns in human pregnancies are associated with preterm delivery. J Nutr. 2014; 144(7): 1075-80.

(19) Institute of Medicine (US) Committee on Improving Birth Outcomes. Reducing Birth Defects: Meeting the Challenge in the Developing World. Bale JR, Stoll BJ, Lucas AO, editores. Washington, D.C.: National Academies Press (US); 2003.

(20) Homocysteine Lowering Trialists' Collaboration. Lowering blood homocysteine with folic acid based supplements: metaanalysis of randomised trials. BMJ. 1998; 316(7135): 894-8.

(21) Brito A, Hertrampf E, Olivares M, Gaitán D, Sánchez $H$, Allen $\mathrm{LH}$, et al. Folatos y vitamina B12 en la salud humana. Rev Med Chil. 2012; 140(11): 1464-75.

(22) Zappacosta B, Mastroiacovo P, Persichilli S, Pounis G, Ruggeri $S$, Minucci $A$, et al. Homocysteine lowering by folate-rich diet or pharmacological supplementations in subjects with moderate hyperhomocysteinemia. Nutrients. 2013; 5(5): 1531-43.

(23) Bukowski R, Malone FD, Porter FT, Nyberg DA, Comstock CH, Hankins GDV, et al. Preconceptional folate supplementation and the risk of spontaneous preterm birth: a cohort study. PLoS Med. 2009; 6(5): e1000061.

(24) Timmermans S, Jaddoe VWV, Hofman A, Steegers-Theunissen
RPM, Steegers EAP. Periconception folic acid supplementation, fetal growth and the risks of low birth weight and preterm birth: the Generation R Study. Br J Nutr. 2009; 102(5): 777-85.

(25) Li X, Li S, Mu D, Liu Z, Li Y, Lin Y, et al. The association between periconceptional folic acid supplementation and congenital heart defects: a case-control study in China. Prev Med. 2013; 56(6): 385-9.

(26) Bower C, Miller M, Payne J, Serna P. Folate intake and the primary prevention of non-neural birth defects. Aust N Z J Public Health. 2006; 30(3): 258-61.

(27) Gaskins AJ, Rich-Edwards JW, Hauser R, Williams PL, Gillman MW, Ginsburg ES, et al. Maternal prepregnancy folate intake and risk of spontaneous abortion and stillbirth. Obstet Gynecol. 2014; 124(1): 23-31.

(28) Obeid R, Koletzko B, Pietrzik K. Critical evaluation of lowering the recommended dietary intake of folate. Clin Nutr. 2014; 33(2): 252-9.

(29) World Health Organization, editor. Prevention of neural tube defects. En: Standards for Maternal and Neonatal Care [Internet]. Génova, Suiza: WHO; 2007. p. 45-8. Recuperado a partir de: http://www.who.int/reproductivehealth/ publications/maternal_perinatal_health/neural_tube_defects. pdf

(30) De-Regil LM, Peña-Rosas JP, Fernández-Gaxiola AC, RaycoSolon P. Effects and safety of periconceptional oral folate supplementation for preventing birth defects. Cochrane Database Syst Rev. 2015; 12: CD007950.

(31) Chaffee BW, King JC. Effect of zinc supplementation on pregnancy and infant outcomes: a systematic review. Paediatr Perinat Epidemiol. 2012; 26 Suppl 1: 118-37.

(32) Tian X, Diaz FJ. Acute dietary zinc deficiency before conception compromises oocyte epigenetic programming and disrupts embryonic development. Dev Biol. 2013; 376(1): 51-61.

(33) Tian X, Diaz FJ. Zinc depletion causes multiple defects in ovarian function during the periovulatory period in mice. Endocrinology. 2012; 153(2): 873-86.

(34) Kim AM, Bernhardt ML, Kong BY, Ahn RW, Vogt S, Woodruff TK, et al. Zinc sparks are triggered by fertilization and facilitate cell cycle resumption in mammalian eggs. ACS Chem Biol. 2011; 6(7): 716-23.

(35) Picco SJ, Anchordoquy JM, de Matos DG, Anchordoquy JP, Seoane A, Mattioli GA, et al. Effect of increasing zinc sulphate concentration during in vitro maturation of bovine oocytes. Theriogenology. 2010; 74(7): 1141-8.

(36) Dickerson EH, Sathyapalan T, Knight R, Maguiness SM, Killick $S R$, Robinson J, et al. Endocrine disruptor \& nutritional effects of heavy metals in ovarian hyperstimulation. ] Assist Reprod Genet. 2011; 28(12): 1223-8.

(37) Tamura T, Goldenberg RL, Johnston KE, DuBard M. Maternal plasma zinc concentrations and pregnancy outcome. Am J Clin Nutr. 2000; 71(1): 109-13.

(38) Saaka M. Combined iron and zinc supplementation improves 
haematologic status of pregnant women in Upper West Region of Ghana. Ghana Med J. 2012; 46(4): 225-33.

(39) Velie EM, Block G, Shaw GM, Samuels S], Schaffer DM, Kulldorff M. Maternal supplemental and dietary zinc intake and the occurrence of neural tube defects in California. Am J Epidemiol. 1999; 150(6): 605-16.

(40) Grajecki D, Zyriax B-C, Buhling KJ. The effect of micronutrient supplements on female fertility: a systematic review. Arch Gynecol Obstet. 2012; 285(5): 1463-71.

(41) Casanueva E, Pfeffer F, Drijanski A, Fernández-Gaxiola AC, Gutiérrez-Valenzuela $V$, Rothenberg $S$ J. Iron and folate status before pregnancy and anemia during pregnancy. Ann Nutr Metab. 2003; 47(2): 60-3.

(42) Ronnenberg AG, Wood RJ, Wang X, Xing H, Chen C, Chen D, et al. Preconception hemoglobin and ferritin concentrations are associated with pregnancy outcome in a prospective cohort of Chinese women. J Nutr. 2004; 134(10): 2586-91.

(43) de Weerd S, Steegers-Theunissen RPM, de Boo TM, Thomas CMG, Steegers E a. P. Maternal periconceptional biochemical and hematological parameters, vitamin profiles and pregnancy outcome. Eur ] Clin Nutr. 2003; 57(9): 1128-34.

(44) Krapels IPC, van Rooij IALM, Ocké MC, West CE, van der Horst CMAM, Steegers-Theunissen RPM. Maternal nutritional status and the risk for orofacial cleft offspring in humans. J Nutr. 2004; 134(11): 3106-13.

(45) Groenen PMW, van Rooij IALM, Peer PGM, Ocké MC, Zielhuis GA, Steegers-Theunissen RPM. Low maternal dietary intakes of iron, magnesium, and niacin are associated with spina bifida in the offspring. J Nutr. 2004; 134(6): 1516-22.

(46) Qiu C, Zhang C, Gelaye B, Enquobahrie DA, Frederick 10, Williams MA. Gestational diabetes mellitus in relation to maternal dietary heme iron and nonheme iron intake. Diabetes Care. 2011; 34(7): 1564-9.

(47) Bao W, Bowers K, Tobias DK, Olsen SF, Chavarro J, Vaag A, et al. Prepregnancy low-carbohydrate dietary pattern and risk of gestational diabetes mellitus: a prospective cohort study. Am ] Clin Nutr. 2014; 99(6): 1378-84.

(48) Bao W, Tobias DK, Olsen SF, Zhang C. Pre-pregnancy fried food consumption and the risk of gestational diabetes mellitus: a prospective cohort study. Diabetologia. 2014; 57(12): 2485-91.

(49) Ford JH. Preconception risk factors and SGA babies: Papilloma virus, omega 3 and fat soluble vitamin deficiencies. Early Hum Dev. 2011; 87(12): 785-9.

(50) Hammiche F, Vujkovic M, Wijburg W, de Vries JHM, Macklon NS, Laven JSE, et al. Increased preconception omega-3 polyunsaturated fatty acid intake improves embryo morphology. Fertil Steril. 2011; 95(5): 1820-3.

(51) Hösli I, Zanetti-Daellenbach R, Holzgreve W, Lapaire O. Role of omega 3-fatty acids and multivitamins in gestation. J Perinat Med. 2007; 35(Suppl 1): S19-24.

(52) Ronnenberg AG, Goldman MB, Chen D, Aitken IW, Willett WC, Selhub J, et al. Preconception homocysteine and B vitamin status and birth outcomes in Chinese women. Am ] Clin Nutr. 2002; 76(6): 1385-91.

(53) Ronnenberg AG, Venners SA, Xu X, Chen C, Wang L, Guang $W$, et al. Preconception B-vitamin and homocysteine status, conception, and early pregnancy loss. Am J Epidemiol. 2007; 166(3): 304-12.

(54) Delange $F$. The role of iodine in brain development. Proc Nutr Soc. 2000; 59(1): 75-9.

(55) Sarici D, Akin MA, Kurtoglu S, Akin L, Tucer B, Yikilmaz A, et al. lodine deficiency: a probable cause of neural tube defect. Childs Nerv Syst. 2013; 29(6): 1027-30.

(56) Zhou SJ, Anderson AJ, Gibson RA, Makrides M. Effect of iodine supplementation in pregnancy on child development and other clinical outcomes: a systematic review of randomized controlled trials. Am ] Clin Nutr. 2013; 98(5): 1241-54.

(57) Moleti M, Di Bella B, Giorgianni G, Mancuso A, De Vivo A, Alibrandi $A$, et al. Maternal thyroid function in different conditions of iodine nutrition in pregnant women exposed to mild-moderate iodine deficiency: an observational study. Clin Endocrinol (Oxf). 2011; 74(6): 762-8.

(58) Donnay S, Arena J, Lucas A, Velasco I, Ares S, Working Group on Disorders Related to lodine Deficiency and Thyroid Dysfunction of the Spanish Society of Endocrinology and Nutrition. Iodine supplementation during pregnancy and lactation. Position statement of the working group on disorders related to iodine deficiency and thyroid dysfunction of the Spanish Society of Endocrinology and Nutrition. Endocrinol Nutr. 2014; 61(1): $27-$ 34.

(59) Ronnenberg AG, Wang X, Xing H, Chen C, Chen D, Guang W, et al. Low preconception body mass index is associated with birth outcome in a prospective cohort of Chinese women. ] Nutr. 2003; 133(11): 3449-55.

(60) Rayco-Solon P, Fulford AJ, Prentice AM. Maternal preconceptional weight and gestational length. Am J Obstet Gynecol. 2005; 192(4): 1133-6.

(61) Ota E, Haruna M, Suzuki M, Anh DD, Tho LH, Tam NTT, et al. Maternal body mass index and gestational weight gain and their association with perinatal outcomes in Viet Nam. Bull World Health Organ. 2011; 89(2): 127-36.

(62) Han YS, Ha EH, Park HS, Kim Y], Lee SS. Relationships between pregnancy outcomes, biochemical markers and pre-pregnancy body mass index. Int J Obes. 2011; 35(4): 570-7.

(63) Gaskins AJ, Rich-Edwards JW, Colaci DS, Afeiche MC, Toth TL, Gillman MW, et al. Prepregnancy and early adulthood body mass index and adult weight change in relation to fetal loss. Obstet Gynecol. 2014; 124(4): 662-9.

(64) Liu X, Chen Q, Tsai H-J, Wang G, Hong X, Zhou Y, et al. Maternal preconception body mass index and offspring cord blood DNA methylation: exploration of early life origins of disease. Environ Mol Mutagen. 2014; 55(3): 223-30.

(65) Willett WC, Howe GR, Kushi LH. Adjustment for total energy intake in epidemiologic studies. Am J Clin Nutr. 1997; 65(4 
Rev Esp Nutr Hum Diet. 2016; 20(1): 48 - 60

La nutrición en el periodo preconcepcional y los resultados del embarazo: revisión bibliográfica y propuesta de intervención del Dietista-Nutricionista

Suppl): 1220 - 1228S; discussion 1229S - 1231S.

(66) Federación Española de Sociedades de Nutrición, Alimentación y Dietética. Ingestas Dietéticas de Referencia (IDR) para la Población Española. 1a. ed. Pamplona, España: EUNSA; 2010.
(67) Nohr EA, Olsen J, Bech BH, Bodnar LM, Olsen SF, Catov JM. Periconceptional intake of vitamins and fetal death: a cohort study on multivitamins and folate. Int J Epidemiol. 2014; 43(1): 174-84. 